\title{
THE FIRST BOUNDARY VALUE PROBLEM FOR THE VIBRATING STRING EQUATION IN DOMAINS WITH PIECEWISE SMOOTH BOUNDARY
}

\author{
A. A. LYASHENKO
}

Institute of Mathematics, Russian Academy of Sciences, Siberian Branch

Universitetskiu Prosp. 4, 630090 Novosibirsk, Russia

0. We investigate the solvability in $\mathbf{W}_{2}^{1}(\Omega)$ of the boundary value problem

$$
\begin{aligned}
& u_{x y}=f, \quad f \in \mathbf{L}_{2}(\Omega),(x, y) \in \Omega, \\
& \left.u\right|_{\partial \Omega}=\varphi(x, y), \quad(x, y) \in \partial \Omega,
\end{aligned}
$$

where $\Omega \subset \mathbb{R}^{2}$ is a convex bounded domain with piecewise smooth boundary $\Gamma=\bigcup_{j=1}^{n} \Gamma_{j}, \Gamma_{j} \subset \mathbf{C}^{2}$. Assume that each $\Gamma_{j}$ is either part of a straight line, or has strictly positive curvature at each point of $\Gamma_{j}$. Also, $\Gamma$ is assumed to have at most two common points with every line parallel to a coordinate axis.

This problem was first studied by J. Hadamard [2]. He noted that it is not well-posed. The history and references can be found in $[1,3,5]$.

We shall look for a solution $u(x, y)$ in the form

$$
u(x, y)=\int_{0}^{x} \int_{0}^{y} f(\xi, \eta) d \xi d \eta+v(x, y)
$$

$(f(x, y)=0$ for every $(x, y) \notin \Omega)$. Then the problem (1), (2) can be written in the form

$$
\begin{array}{ll}
v_{x y}=0, & (x, y) \in \Omega, \\
v=h(x, y), & (x, y) \in \partial \Omega .
\end{array}
$$

It is well known that every generalized solution $v \in \mathbf{W}_{2}^{1}(\Omega)$ of (3) can be written in the form

$$
v(x, y)=p(x)+q(y)
$$

where $p, q$ are arbitrary functions in $\mathbf{W}_{2}^{1}(\Omega)$. So the problem (3), (4) can be 
reformulated as follows: find $p, q \in \mathbf{W}_{2}^{1}(\Omega)$ which satisfy

$$
p(x)+q(y)=h(x, y), \quad(x, y) \in \partial \Omega .
$$

Following [3], we call the points of $\Gamma$ at which there is a line of support parallel to a coordinate axis the vertices of $\Gamma$. It is evident that $\Gamma$ has either two, three or four vertices.

We shall consider here the cases of two and three vertices. In those cases, necessary and sufficient conditions for the solvability of the problem (3), (4) in $\mathbf{W}_{2}^{1}(\Omega)$ and explicit formulae for solutions will be obtained. Uniqueness of solutions was proved in [4].

1. Let $\Gamma$ have two vertices $P_{0}, P_{1}$. We shall consider a regular case: the onesided tangents to $\Gamma$ at $P_{0}, P_{1}$ are not parallel to coordinate axes. Let $s$ be a natural parametrization of $\Gamma: \Gamma=\{(x(s), y(s)) \mid 0 \leq s<l\},(x(0), y(0))=P_{0}$, $\left(x\left(s_{1}\right), y\left(s_{1}\right)\right)=P_{1}$. Define

$$
\begin{gathered}
H(s)=h(x(s), y(s)), \\
P(s)=p(x(s)), \quad Q(s)=q(y(s)) .
\end{gathered}
$$

It is not hard to prove the following statement.

Lemma 1.1. $p, q \in \mathbf{W}_{2}^{1}(\Omega)$ if and only if $P, Q \in \mathbf{W}_{2, \varrho}^{1}(0, l)$, where $\varrho(s)=$ $s(l-s)\left|s_{1}-s\right|, s \in(0, l)$.

Define onto functions $f^{+}, f^{-}:(0, l) \rightarrow(0, l)$ to be the nontrivial solutions of the equations

$$
x(s)=x\left(f^{+}(s)\right), \quad y(s)=y\left(f^{-}(s)\right), \quad s \in(0, l) .
$$

Then $P, Q$ defined by (7) satisfy

$$
P(s)=P\left(f^{+}(s)\right), \quad Q(s)=Q\left(f^{-}(s)\right), \quad s \in(0, l) .
$$

So, the problem (3), (4) can be reformulated as follows: find $P, Q \in \mathbf{W}_{2, \varrho}^{1}(0, l)$ satisfying (9) and

$$
P(s)+Q(s)=H(s), \quad s \in(0, l) .
$$

Define onto functions $f_{k}:(0, l) \rightarrow(0, l), k \in \mathbb{Z}$, by

$$
f_{0}(s)=s, \quad f_{1}(s)=f^{-}\left(f^{+}(s)\right), \quad f_{-1}(s)=f^{+}\left(f^{-}(s)\right), \quad f_{k+1}(s)=f_{1}\left(f_{k}(s)\right) .
$$

The properties of the functions $f_{k}$ were described in [4]. Choose $\psi_{0} \in\left(0, s_{1}\right)$ and define a system of intervals $M_{k}, k \in \mathbb{Z}$, by

$$
\begin{gathered}
M_{0}= \begin{cases}\left(f_{1}\left(\psi_{0}\right), \psi_{0}\right], & f_{1}\left(\psi_{0}\right)<\psi_{0}, \\
\left(\psi_{0}, f_{1}\left(\psi_{0}\right)\right], & f_{1}\left(\psi_{0}\right)>\psi_{0},\end{cases} \\
M_{2 k}=\left\{f_{k}(s) \mid s \in M_{0}\right\}, \quad M_{2 k+1}=\left[f^{+}\left(f_{k}(s)\right) \mid s \in M_{0}\right\} .
\end{gathered}
$$

The following lemma was proved in [4].

LEMma 1.2. 1) $M_{k} \cap M_{m}=\emptyset, k \in \mathbb{Z}, k \neq m$.

2) $\bigcup_{k=-\infty}^{+\infty} M_{2 k}=\left(0, s_{1}\right), \bigcup_{k=-\infty}^{\infty} M_{2 k+1}=\left(s_{1}, l\right)$. 
Define $w(s)$ for $s \in(0, l) \backslash\left\{s_{1}\right\}$ as follows: if $s \in\left(0, s_{1}\right)$, then

$$
w(s)= \begin{cases}0, \quad s \in M_{0}, & \\ \sum_{j=-k+1}^{0} H\left(f_{j}(s)\right)-H\left(f^{-}\left(f_{j}(s)\right)\right), & s \in M_{2 k}, k \in \mathbb{N}, \\ -\sum_{j=1}^{k} H\left(f_{j}(s)\right)-H\left(f^{-}\left(f_{j}(s)\right)\right), & s \in M_{-2 k}, k \in \mathbb{N}\end{cases}
$$

if $s \in\left(s_{1}, l\right)$, then

$$
w(s)=w\left(f^{+}(s)\right) .
$$

The following theorem is the main result of this section.

THEOREM 1.1. There exist $P, Q \in \mathbf{W}_{2, \varrho}^{1}(0, l)$ satisfying $(9),(10)$ if and only if the following conditions hold:

1) for all $s \in\left(0, s_{1}\right)$

(a) $w\left(f_{k}(s)\right)-w\left(f_{-k}(s)\right)-w\left(f_{k}\left(\psi_{0}\right)\right)+w\left(f_{-k}\left(\psi_{0}\right)\right) \rightarrow 0$ as $k \rightarrow \infty$;

(b) $w\left(f_{k}(s)\right)-w\left(f_{k+1}(s)\right) \rightarrow 0$ as $k \rightarrow-\infty$;

2) (a) for all $s \in(0, l) \backslash\left\{s_{1}\right\}$ the following limits exist:

$$
w_{1}(s)= \begin{cases}\lim _{k \rightarrow \infty}\left(w\left(f_{k}(s)\right)-w\left(f_{k}\left(\psi_{0}\right)\right)\right), & s \in\left(0, s_{1}\right), \\ \lim _{k \rightarrow \infty}\left(w\left(f_{k}(s)\right)-w\left(f_{k}\left(f^{+}\left(\psi_{0}\right)\right)\right)\right), & s \in\left(s_{1}, l\right)\end{cases}
$$

(b) $w-w_{1} \in \mathbf{W}_{2, \varrho}^{1}(0, l), H-w+w_{1} \in \mathbf{W}_{2, \varrho}^{1}(0, l)$.

If conditions 1$), 2)$ hold, then all solutions $P, Q \in \mathbf{W}_{2, \varrho}^{1}(0, l)$ of $(9),(10)$ have the form

$$
P(s)=w(s)-w_{1}(s)+c, \quad Q(s)=H(s)-w(s)+w_{1}(s)-c, \quad c \in \mathbb{R} .
$$

2. Let $\Gamma$ have three vertices $P_{0}, P_{1}, P_{2}$. Moreover, suppose that neither of the lines $x=$ const, $y=$ const through $P_{0}$ intersects $\Omega$, and the line $x=$ const (resp. $y=$ const) through $P_{1}$ (resp. $P_{2}$ ) does not meet $\Omega$. We shall consider a regular case: the one-sided tangents to $\Gamma$ at $P_{0}$ are not parallel to coordinate axes. Let $s$ be a natural parametrization of $\Gamma: \Gamma=\{(x(s), y(s)) \mid 0 \leq s<l\},(x(0), y(0))=P_{0}$, $\left(x\left(s_{j}\right), y\left(s_{j}\right)\right)=P_{j}, j=1,2$, where $s_{1}, s_{2} \in(0, l)$.

Define $\mu_{j}^{ \pm} \in \mathbb{R} \cup\{ \pm \infty\}, j=1,2$, by

$$
\mu_{j}^{ \pm}=\lim _{s \rightarrow s_{j} \pm 0} y^{\prime}(s) / x^{\prime}(s), \quad j=1,2 .
$$

Define $d_{j}(s), \varrho_{j}(s), j=1,2, s \in(0, l)$, by

$$
\begin{aligned}
& d_{j}(s)= \begin{cases}1, & \mu_{j}^{+}=\mu_{j}^{-}, \\
\left|s_{j}-s\right|, & \mu_{j}^{+} \neq \mu_{j}^{-}, 0<\left|\mu_{j}^{ \pm}\right|<\infty, \\
\sqrt{\left|s_{j}-s\right|}+\theta\left(s-s_{j}\right), & 0<\left|\mu_{j}^{-}\right|<\infty,\left|\mu_{j}^{+}\right| \in\{0, \infty\}, \\
\sqrt{\left|s_{j}-s\right|}+\theta\left(s_{j}-s\right), & 0<\left|\mu_{j}^{+}\right|<\infty,\left|\mu_{j}^{-}\right| \in\{0, \infty\},\end{cases} \\
& \varrho_{j}(s)=s(l-s) d_{j}(s),
\end{aligned}
$$


where $\theta(s)=(1+\operatorname{sign}(s)) / 2$.

It is not hard to prove the following result.

Lemma 2.1. $p \in \mathbf{W}_{2}^{1}(\Omega)$ (resp. $q \in \mathbf{W}_{2}^{1}(\Omega)$ ) if and only if

$$
P(s)=p(x(s)) \in \mathbf{W}_{2, \varrho_{2}}^{1}(0, l) \quad\left(\text { resp. } Q(s)=q(y(s)) \in \mathbf{W}_{2, \varrho_{1}}^{1}(0, l)\right) .
$$

So, the problem (3), (4) in this case can be reformulated as follows: find $P \in \mathbf{W}_{2, \varrho_{2}}^{1}(0, l), Q \in \mathbf{W}_{2, \varrho_{1}}^{1}(0, l)$ satisfying (9), (10) for all $s \in(0, l) \backslash\left\{s_{1}, s_{2}\right\}$.

Define a system of open intervals $M_{k}, k \in \mathbb{Z}$, by

$$
\begin{aligned}
M_{0} & = \begin{cases}\left(s_{1}, s_{2}\right), & s_{1}<s_{2}, \\
\left(s_{2}, s_{1}\right), & s_{1}>s_{2},\end{cases} \\
M_{2 k} & =\left\{f_{k}(s) \mid s \in M_{0}\right\}, \quad M_{2 k+1}=\left\{f^{+}\left(f_{k}(s)\right) \mid s \in M_{0}\right\} .
\end{aligned}
$$

The following lemma was proved in [4].

LEMma 2.2. 1) $M_{k} \cap M_{m}=\emptyset, k, m \in \mathbb{Z}, k \neq m$.

2) $\bigcup_{k=-\infty}^{\infty} \bar{M}_{k}=(0, l)$

Define $w(s)$ for $s \in \bigcup_{k=-\infty}^{\infty} M_{k}$ by formula (11) if $s \in \bigcup_{k=-\infty}^{\infty} M_{2 k}$, and by (12) if $s \in \bigcup_{k=-\infty}^{\infty} M_{2 k+1}$.

The following theorem is the main result of this section.

Theorem 2.1. There exist $P \in \mathbf{W}_{2, \varrho_{2}}^{1}(0, l), Q \in \mathbf{W}_{2, \varrho_{1}}^{1}(0, l)$ satisfying (9), (10) for all $s \in(0, l) \backslash\left\{s_{1}, s_{2}\right\}$ if and only if the following conditions hold:

1) for all $s \in \bigcup_{k=-\infty}^{\infty} M_{k}$,

(a) $w\left(f_{k}(s)\right)-w\left(f_{-k}(s)\right) \rightarrow 0$ as $k \rightarrow \infty$;

(b) $w\left(f_{k}(s)\right)-w\left(f_{k+1}(s)\right) \rightarrow 0$ as $k \rightarrow-\infty$;

2) (a) for all $s \in \bigcup_{k=-\infty}^{\infty} M_{k}$ the limit

$$
w_{1}(s)=\lim _{k \rightarrow \infty}\left(w\left(f_{k}(s)\right)-w\left(f_{k}\left(\psi_{0}\right)\right)\right)
$$

exists, where $\psi_{0} \in M_{0}$ is arbitrary;

(b) $\left(w(s)-w_{1}(s)\right) \in \mathbf{W}_{2, \varrho_{2}}^{1}(0, l)$;

$$
\left(H(s)-w(s)+w_{1}(s)\right) \in \mathbf{W}_{2, \varrho_{1}}^{1}(0, l) .
$$

If conditions 1 ), 2) hold, then all solutions $P \in \mathbf{W}_{2, \varrho_{2}}^{1}, Q \in \mathbf{W}_{2, \varrho_{1}}^{1}$ of (9), (10) have the form (14).

Re mark 1. Conditions 1), 2) of Theorems 1.1, 2.1 are in fact the conditions of orthogonality of the right sides of (1), (2) to $\mathbf{L}_{2}(\Omega)$ generalized solutions of the homogeneous problem (1), (2).

R e m a r k 2. Irregular cases for two and three vertices can be handled similarly.

Remark 3. Consider the family of differential operators $L_{\lambda} u=(1-\lambda) u_{x x}-$ $\lambda u_{y y}, \lambda \in(0,1)$. Then there are bounded convex domains with piecewise smooth boundary such that for every $\lambda \in(0,1)$ except some set $\left\{\lambda_{1}, \ldots, \lambda_{k}\right\}$ the boundary has less than four vertices in the coordinates $\xi=\sqrt{\lambda} \cdot x+\sqrt{1-\lambda} \cdot y, \eta=$ $\sqrt{\lambda} \cdot x-\sqrt{1-\lambda} \cdot y$. A general form of such domains was described in [4]. 


\section{References}

[1] R. A. Aleksandryan, The spectral properties of the operator generated by a system of differential equations of S. L. Sobolev's type, Trudy Moskov. Mat. Obshch. 9 (1960), 455505 (in Russian).

[2] J. Hadamard, On some topics connected with linear partial differential equations, Proc. Benares Math. Soc. 3 (1921), 39-48.

[3] F. John, The Dirichlet problem for a hyperbolic equation, Amer. J. Math. 63 (1) (1941), 141-154.

[4] A. A. Lyashenko, On a problem of S. L. Sobolev in domains with corners, preprint No. 27, Institute of Mathematics, Sib. Branch Acad. of Sci. of USSR, Novosibirsk 1987, 22 pp. (in Russian).

[5] B. D. Sleeman, Boundary value problems for the vibrating string equation, Proc. Roy. Soc. Edinburgh Ser. A 88 (1-2) (1981), 185-194. 